\title{
Sirt-1 as A Predictive Marker of Metabolic Syndrome in Psoriasis Patients
}

H.H.sabry ${ }^{1}$, A.M.Hamed ${ }^{1}$, A.A.El Fallah ${ }^{2}$ and A.R.Ghonamey ${ }^{3}$

${ }^{1}$ Dermatology, Venereology and Andrology Dept., Faculty of Medicine, Benha Univ., Benha, Egypt

${ }^{2}$ Clinical and Chemical Pathology Dept., Faculty of Medicine, Benha Univ., Benha, Egypt

${ }^{3}$ Dermatology,Venereology and Andrology Dept., Al.Houd Almarsoud hospital, Cairo, Egypt

E.Mail:dr.amiraghonamey@gmail.com

\begin{abstract}
Psoriasis affects mainly skin, nails and joint and is characterized by the presence of sharply demarcated, red plaques with adherent silvery white scales and a tendency for symmetrical distribution over the body. Psoriasis can be provoked by external and internal triggers, including mild trauma, sunburn, infections, systemic drugs and stress. There is a strong positive association between psoriasis and known risk factors including central obesity, insulin resistance and dyslipidemia. Sirt-1 is a NAD+-dependent deacetylase involved in the regulation of a myriad of cellular processes including mitochondrial biogenesis, genomic stability, cellular senescence and apoptosisAim: The aim of this study was to assess serum levels of sirt-1 in psoriasis patients and correlate their levels with the severity of the disease.. Method: This present study included: Patient group (group 1) included 25 psoriasis patients. In addition, 15 apparently healthy, age and sex matched individuals included as a control group (Group 2). All were investigated for serum sirt 1 level and compared. Serum Sirt 1 were estimated using commercially available ELISA kits. Conclusion: From the results of present study, it is concluded that sirt 1levels are lower in psoriasis patients not corrospnding to the disease severity.
\end{abstract}

Keywords: Psoriasis, Metabolic syndrome, Sirt 1.

\section{Introduction}

Psoriasis affects mainly skin, nails and joint and is characterized by the presence of sharply demarcated, red plaques with adherent silvery white scales and a tendency for symmetrical distribution over the body [1].

The worldwide prevalence of psoriasis is approximately $3 \%$ of all cutaneous diseases [2]. There is a study of 2194 children in Egypt, the prevalence of psoriasis among people 18 years of age and younger was $0.05 \%[3]$.

Psoriasis has a bimodal age of disease onset. The first peak is around 20 years and the second peak is around 60 years. People with disease onset around 20 years old have stronger genetic predisposition, they have a higher prevalence of having HLA-Cw6. The linkage to genetic factor is lower for the group with late onset disease [4].

The etiology of psoriasis remains unclear, although there is evidence for genetic predisposition [5]. Although there is a suggestion that psoriasis could be an autoimmune disease, no autoantigen that could be responsible has been defined yet. Psoriasis can also be provoked by external and internal triggers, including mild trauma, sunburn, infections, systemic drugs and stress [6].

Metabolic syndrome (MetS) represents a cluster of metabolic abnormalities that include hypertension, central obesity, insulin resistance and atherogenic dyslipidemia, [7].

There is a strong positive association between psoriasis and central obesity, insulin resistance and dyslipidemia has been described, [8].

Sirtuin 1 (Sirt-1) is a NAD+-dependent deacetylase involved in the regulation of a myriad of cellular processes including mitochondrial biogenesis, genomic stability, cellular senescence and apoptosis [9].

\section{Materials and methods \\ 2.1The Study population}

This is a case-control study .The current study had been conducted between april 2019 and October 2019 after the approval by Research Committee at Faculty of Medicine, Benha University. The study included 40 subjects that were randomly selected from those attending the Dermatology Outpatient's Clinic at Benha University Hospital, Faculty of Medicine, Benha University, Qalyobia, Egypt. Prior to initiation of the study, every subject was informed about the aim of the study and an informed consent was obtained from each individual before sample collection.This present study included: Patient group (group 1) included 25 psoriasis patients. In addition, 15 apparently healthy, age and sex matched individuals included as a control group(Group2).

\subsection{ELISA assays of sirt 1}

Quantitative determinations of serum sirt1 were achieved using a double-

Antibody sand wich Enzyme Linked Immune Sorbent Assay technique (ELISA) was used to detect serum level of sirt1 using a commercial Human sirt1 ELISA Kit for research use only (Cat \#: 201-12-8108, SunRedBio, China).

\subsection{Statistical analysis}

- The collected data was revised, coded, tabulated and introduced to a PC using Statistical package for Social Science (IBM Corp. Released 2011. IBM SPSS Statistics for Windows, Version 20.0. Armonk, NY: IBM Corp.). Data were presented and suitable analysis was done according to the type of data obtained for 
- $\quad$ each parameter.Student T Test was used to assess the statistical significance of the difference between two study group means.For the comparison of the three groups' means, one way analysis of variance (ANOVA) was used. Chi-Square test was used to examine the relationship between two qualitative variables. Fisher's exact test: was used to examine the relationship between two qualitative variables when the expected count is less than 5 in more than $20 \%$ of cells.Correlation analysis: To assess the strength of association between two quantitative variables. The correlation coefficient defines the strength and direction of the linear relationship between two variables. $\mathrm{p}$ is significant if $<0.05$ at confidence interval $95 \%$.

\section{Results}

The duration of psoriasis patients in patient group ranged between 2-7 years and their PASI score ranged between2-8 Table (1).

Table (1) Clinical data among patients.

\begin{tabular}{lcc}
\hline & Rang & Mean \pm SD \\
\hline Duration & $2-7$ & $3.4 \pm 2.43$ \\
PASI Score & $2-8$ & $3.7 \pm 2.9$ \\
\hline
\end{tabular}

\subsection{Biochemical Assessments of Sirt 1}

The mean sirt-1 serum levels were significantly lower in patient group than their controls Table (2) .

Table (2) Comparison between patient group and their controls regarding sirt-l (ng/dl).

\begin{tabular}{lccccc}
\hline & & Group 1 & Group 2 & t. test & P. value \\
\hline $\begin{array}{l}\text { Sirt l } \\
(\text { ng/dl) }\end{array}$ & Range & $2.83-5.82$ & $7.2-11.5$ & & \\
& Mean \pm SD & $3.88 \pm 1.2$ & $8.22 \pm 1.3$ & 12.51 & $<0.05$ \\
\hline
\end{tabular}

There was statistically significant negative correlation between sirt-1 (ng/dl) and TG (mg/dl), and and there was no statistically significant correlations between sirt-1 (ng/dl) and HDL, FBS (mg/dl), waist circumference (cm), SBP, DBP, age, duration of the disease and PASI score Table (3).

Table (3) Correlations of sirt-l (ng/dl) in Patients group.

\begin{tabular}{lcc}
\hline \multirow{2}{*}{ Correlation } & \multicolumn{2}{c}{ Sirt 1 (mg/dl) } \\
\cline { 2 - 3 } & $\mathbf{R}$ & $\mathbf{p}$ \\
\hline Age (Years) & .33 & .12 \\
Duration & .07 & .77 \\
PASI Score & .09 & .54 \\
FBS(mg/dl) & -.71 & 0.3 \\
TG (mg/dl) & -.82 & 0.05 \\
HDL (mg/dl) & .52 & 0.07 \\
Waist Circumference (cm) * & .82 & 0.08 \\
SBP & $.92-$ & 0.2 \\
DBP & $.53-$ & 1.2 \\
\hline
\end{tabular}

\section{Discussion}

This case-controlled study was designed to assess serum levels of sirt-1 in psoriasis patients and correlate their levels with the severity of the disease.

In the current study, there were statistically significant decrease in sirt- 1 (ng/dl) level among patients group and their controls,

This was in agreement with a study that observed that, sirt-1 was significantly lower in lesional skin [10].

In our study, there was no correlation between sirt-1 and age, duration of psoriasis and PASI score in psoriasis patients. From our point of view, absence of correlation with age and duration referred to low mean age and duration of selected patients.
Regarding to the age, [11] illustrated that patients with MetS had a higher mean age and earlier age of onset of psoriasis.

Regarding to the duration, [12] reported that, there was no significant difference observed for occurrence of MetS with respect to longer or shorter duration of psoriasis.

Regarding to PASI score, these results agreed with several studies conducted by [13,14,15 and 12] showed that, MetS is present irrespective of severity of psoriasis.

Also, D.Sommer [16] reported that, MetS prevalence as $30 \%$ in hospitalized psoriasis population whose mean PASI score was 11. These data indicate that MetS prevalence may be associated with psoriasis severity [17].

Furthermore, M.Tupikowska-Marzec [18] found that, there is a strong association between MetS and psoriatic 
diseases, regardless of psoriasis severity or casualty. Other studies are establishing the association of the increase of BMI, hip and waist circumference, and insulin concentration (which are the main components in MetS) with the severity of psoriasis .

\section{Conclusions}

From the results of present study, it is concluded that sirt 1levels are lower in psoriasis patients not corrospnding to the disease severity .

\section{Recommendations}

The results of our study should be interpreted in light of its limitations, as the present study included a relatively small sample size. Further studies are needed to investigate the precise mechanisms by which sirt- 1 contribute to the pathogenesis of both psoriasis and MetS.

\section{Conflict Of Interest} interest.

The authors declare that they have no conflict of

\section{References}

[1] P.Kurizky, C.Kurizky and L.Nogueira, Treatment of psoriasis and psoriatic arthritis during pregnancy and breast feeding. An Bras Dermatol,Vol.90(3),PP.36775,2015 .

[2] G.Yamamah, H.Emam, M.Abdelhamid, M.Elsaie, T.Farid, Epidemiologic study of dermatologic disorders among children in South Sinai, Egypt. Int J Dermatol,Vol.51(10),PP.1180-1185,2012.

[3] H.King-man, Psoriasis. Medical Bulletin,Vol.15,PP.5-12,2010.

[4] J.Harden, J.Krueger and A.Bowcock, The immunogenetics of psoriasis: a comprehensive review. J Autoimmun, Vol.64,PP.66-73,2015.

[5] W.Boehncke and M.Schon, Psoriasis. Lancet,Vol.386(9997),PP.983-994,2015.

[6] E.Ogawa, Y.Sato, A.Minagawa and R.Okuyama, Pathogenesis of psoriasis and development of treatment. J Dermatol,Vol.45(3),PP.264-272,2018.

[7] S.Itani, A.Arabi and D. Harb, High prevalence of metabolic syndrome in patients with psoriasis in Lebanon: a prospective study. Int. J Dermatol,Vol.55(4),PP.390-395,2016.

[8] Y.Yuan, V.Cruzat, P.Newshome, J.Cheng, Y.Chen, Regulation of SIRT1 in aging: roles in mitochondrial function and biogenesis. Mech Ageing Dev,Vol.155,PP.10-21,2016.
[9] H.Rasheed, M.El-Komy, R.Hegazy, H.Gawdat, A.AlOrbani, Expression of sirtuins 1, 6, tumor necrosis factor, and interferon-gamma in psoriatic patients. Int. J Immunopathol Pharmacol,Vol.29,PP.764-768,2016.

[10] E.Lee, J.Han, C.Bang, S.Yoo, K.Han, Risk of EndStage Renal Disease in Psoriatic Patients: Real-World Data from a Nationwide Population-Based Cohort Study. Sci Rep,Vol.9,PP.1-16581,2019.

[11] A.Salunke, M.Nagargoje, V.Belgaumkar, S.Tolat and R.Chavan, Association of Metabolic Syndrome in Chronic Plaque Psoriasis Patients and their Correlation with Disease Severity, Duration and Age: A Case Control Study from Western Maharashtra. J Clin Diagn Res,Vol.11(8),PP.6-10,2017.

[12] S.Madanagobalane and S.Anandan, Prevalence of metabolic syndrome in South Indian patients with psoriasis vulgaris and the relation between disease severity and metabolic syndrome: a hospital-based case-control study. Indian J Dermatol,Vol.57(5),PP.353-357,2012.

[13] S.Lakshmi, A.Kumar and C.Udayashankar, Metabolic syndrome in patients with psoriasis: A comparative study. Indian Dermatol Online J,Vol.5(2),PP.132-137,2014.

[14] M.Morgaonkar, R.Kushwaha, S.Gupta, S.Jain, D.Kulkarni, Prevalence of metabolic syndrome in psoriasis vulgaris: A cross-sectional study from a tertiary care hospital of south-east Rajasthan. Int. J Res Dermatol,Vol.2,PP.30-35,2016.

[15] E.Malkic, N.Hadzigrahic and A.Cickusic, Psoriasis and metabolic syndrome. Med Arch,Vol.69,PP.8587,2015 .

[16] D.Sommer, S.Jenisch, M.Suchan, E.Christophers and M.Weichenthal, Increased prevalence of the metabolic syndrome in patients with moderate to severe psoriasis. Arch Dermatol Res, Vol.298,PP.321$328,2006$.

[17] X.Gui, X.Yu, H.Jin, Y.Zuo and C.Wu, Prevalence of metabolic syndrome in Chinese psoriasis patients: a hospital-based cross-sectional study. J Diabetes Investig,Vol.9,PP.39-43,2018.

[18] M.Tupikowska-Marzec, K.Kolačkov, A.ZdrojowyWełna, N.Słoka, J.Szepietowski, The influence of FTO polymorphism rs9939609 on obesity, some clinical features, and disturbance of carbohydrate metabolism in patients with psoriasis. Biomed Res Int,Vol.7304345,PP.22-25, 2019. 